Revue des sciences de l'eau

\title{
L'expérience algérienne dans le domaine des études de retenues collinaires
}

\section{The Algerian experience in the domain of small dams studies}

\section{B. Benlaoukli et B. Touaïbia}

Volume 17, numéro 2, 2004

URI : https://id.erudit.org/iderudit/705527ar

DOI : https://doi.org/10.7202/705527ar

Aller au sommaire du numéro

Éditeur(s)

Université du Québec - INRS-Eau, Terre et Environnement (INRS-ETE)

ISSN

0992-7158 (imprimé)

1718-8598 (numérique)

Découvrir la revue

Citer cet article

Benlaoukli, B. \& Touaïbia, B. (2004). L'expérience algérienne dans le domaine des études de retenues collinaires. Revue des sciences de l'eau / Journal of Water Science, 17(2), 153-162. https://doi.org/10.7202/705527ar
Résumé de l'article

Nous présentons dans cet article l'expérience algérienne acquise dans le domaine des études de retenues collinaires depuis 1987 à ce jour. En effet, plus de $40 \%$ des retenues collinaires ayant fait l'objet d'une étude sont aujourd'hui en exploitation et ont atteint l'objectif visé.

Avec une demande en eau croissante, l'Algérie s'est vue contrainte de mobiliser au maximum ses ressources en eau superficielles par la réalisation de petits ouvrages.

Après les échecs enregistrés dans la réalisation de tels ouvrages du fait de la négligence d'études préalables, l'administration publique concernée pris en charge sérieusement la conception des retenues collinaires sur la base d'un dossier d'études répondant à des normes techniques établies. Le manque d'expérience dans de telles études a engendré un investissement colossal dans les travaux de conception. Pour remédier à cette situation, les bureaux d'études algériens ont développé leur propre méthodologie d'étude.

Contrairement aux grands barrages, les petits barrages et retenues collinaires ont été considérés différemment vu leurs particularités. La consistance des études a été sensiblement modifiée avec comme résultat des délais de réalisation qui sont passés de 12 à 6 mois se répercutant aussi sur le coût global, qui est passé de 2500000 à 1200000 DA (50 000 à 25000 \$), sans pour autant affecter la qualité et la fiabilité de l'étude. Cette réduction a été obtenue grâce à la normalisation de certaines études et à la rationalisation des tâches, comme l'étude hydrologique, les reconnaissances géologiques et géotechniques (sondages, fouilles, essais en laboratoire), le dimensionnement hydraulique des ouvrages, le calcul de génie civil des ouvrages en béton armé et les pièces dessinées.

La réduction des délais a été obtenue également grâce à la maîtrise technique et à l'outil informatique. Les travaux d'études ont été scindés en six missions distinctes : travaux topographiques, étude hydrologique, étude géologique et géotechnique, étude de faisabilité, étude d'exécution, et dossier d'appel d'offre. En effet l'étude hydrologique ne fait ressortir que les paramètres essentiels nécessaires à la suite des travaux d'études. L'étude géologique et géotechnique, sur la base d'une campagne de reconnaissance restreinte et d'essais en laboratoire limités, définit le type de remblai à mettre en place, les remèdes à prévoir dans le traitement des fondations, et les paramètres géotechniques rentrant dans le dimensionnement des ouvrages.

Le choix du type de remblai qui est lié à la disponibilité des matériaux de construction en quantité et en qualité, se limite à un remblai homogène ou zoné. Les détails du remblai font l'objet d'un plan d'exécution standard valable pour toutes les retenues, il suffit seulement de mettre les cotes correspondantes.

Le choix du type d'évacuateur de crues dépend essentiellement du relief. Il est préconisé des évacuateurs de crues à surface libre à entonnement frontal ou latéral. Les hauteurs des murs bajoyer ont été standardisées et des plans types de ferraillage de différentes hauteurs sont élaborés, prêts pou être adaptés à l'étude considérée. Les bassins d'amortissement qui sont préconisés sont de types USBR. Deux types d'ouvrages de vidange et de prise d'eau ont fait l'objet d'une préparation technique et dimensionnelle, dans le but de les caler sur tous les sites de retenues collinaires, et des plans d'exécution sont élaborés et prêts à une large utilisation.

Pour mieux cerner ces études, un échantillon de plus d'une vingtaine de retenues collinaires réalisées et actuellement en exploitation, dans cinq Wilayas du Nord de l'Algérie, est présenté. La diffusion de éens mérite d'être prise en considération et peut contribuer quelque part à améliorer la sécurité et les performances de ces ouvrages.
Tous droits réservés (C) Revue des sciences de l'eau, 2004
Ce document est protégé par la loi sur le droit d'auteur. L’utilisation des services d’Érudit (y compris la reproduction) est assujettie à sa politique d'utilisation que vous pouvez consulter en ligne.

https://apropos.erudit.org/fr/usagers/politique-dutilisation/ 


\title{
L'expérience algérienne dans le domaine des études de retenues collinaires
}

\author{
The Algerian experience in the domain of small \\ dams studies
}

B. BENLAOUKLI ${ }^{1}$, B. TOUAÏBIA ${ }^{2}$

Reçu le 19 septembre 2002, accepté le 28 mai 2003*.

\section{SUMMARY}

The objective of this paper was to summarize the information obtained from studies of small dams in Algeria from 1987 to the present With the growing consumer demand, Algeria chose to maximize its surface water resources by the construction of small dams. Following the inadequate performance of these projects, the public administration re-evaluated its responsibility for designing small dams. The lack of experience with such studies resulted in a big investment in dam design. To improve this situation, the Algerian consulting engineers developed their own calculation methodology. In contrast to big dams, small dams are unique due to their particular characteristics. The studies have become more consistent and as a result the construction delays have decreased from 12 to 6 months, thus reducing the overall cost, which passed from 2500000 to $1200000 \mathrm{DA}(\$ 50000$ to $\$ 25000$ ) without affecting the quality and the reliability of the design. These improvements have been achieved thanks to a certain normalization of the studies and a rationalization of the tasks, such as the hydrological study, the geological and geotechnical investigations (bores, excavations, laboratory tests), the hydraulic design of the construction works, the calculation of the reinforced concrete works and the drawings.

The reduction in delays was obtained thanks to technical improvements and computing tools. The working studies were divided into six distinct projects: topographical; hydrological; geological and geotechnical; feasibility; planning; and call for tenders. The hydrological study highlights only essential parameters that are required for the continuation of studies. The geological and geotechnical study, performed on the basis of data collected in a reconnaissance campaign and data from limited laboratory tests, defines the type of embankment required, considers the possible treatments for the foundations, and generates the geotechnical parameters needed for scaling the hydraulic structure.

1 PhD Ouvrages Hydrotechniques. Laboratoire d'Hydraulique. École Nationale Supérieure de l'Hydraulique. BP 31.09000. Blida. Algérie. bbenlaoukli@yahoo.fr.

2 Docteur d'État. Maître de Conférences. Laboratoire d'Hydrologie. École Nationale Supérieure de l'Hydraulique. BP 31. 09000. Blida. Algérie. touaibia@yahoo.fr.

* Les commentaires seront reçus jusqu'au 30 décembre 2004. 
The choice of type of embankment, which is linked at the availability of construction materials of suitable quality, is limited to either a homogeneous or zoned embankment. The details of the embankment are derived from the application of a standard execution plan, which is valid for all projects involving small dams; only the local geotechnical levels need to be specified. The choice of spill wall mainly depends on the relief. Two types of spillways are recommended: lateral with frontal entry; and lateral with lateral entry. The heights of the spillway walls are standardized, and plans for an iron framework with different heights are developed, ready to be adapted to the specific project. USBR types stilling basins are recommended. Two standardised outlet types have been selected and designed, to be applied to all the small dam sites, and the implementation plans are developed and ready for a wide use.

To better understand these studies, a sample of more than twenty small dams currently in operation, in five Wilayases in northern Algeria, is presented. Our experiences, although limited when compared to certain countries in the Mediterranean basin, deserve to be taken into consideration and can contribute in improvements in the security and performance of small dams.

Key words: hillside dam, standardised studies, hydraulic scaling, dam design, implementation plans.

Nous présentons dans cet article l'expérience algérienne acquise dans le domaine des études de retenues collinaires depuis 1987 à ce jour. En effet, plus de $40 \%$ des retenues collinaires ayant fait l'objet d'une étude sont aujourd'hui en exploitation et ont atteint l'objectif visé.

Avec une demande en eau croissante, l'Algérie s'est vue contrainte de mobiliser au maximum ses ressources en eau superficielles par la réalisation de petits ouvrages.

Après les échecs enregistrés dans la réalisation de tels ouvrages du fait de la négligence d'études préalables, l'administration publique concernée pris en charge sérieusement la conception des retenues collinaires sur la base d'un dossier d'études répondant à des normes techniques établies.

Le manque d'expérience dans de telles études a engendré un investissement colossal dans les travaux de conception. Pour remédier à cette situation, les bureaux d'études algériens ont développé leur propre méthodologie d'étude. Contrairement aux grands barrages, les petits barrages et retenues collinaires ont été considérés différemment vu leurs particularités. La consistance des études a été sensiblement modifiée avec comme résultat des délais de réalisation qui sont passés de 12 à 6 mois se répercutant aussi sur le cô̂t global, qui est passé de 2500000 à $1200000 \mathrm{DA}(50000$ à $25000 \$)$, sans pour autant affecter la qualité et la fiabilité de l'étude. Cette réduction a été obtenue grâce à la normalisation de certaines études et à la rationalisation des tâches, comme l'étude hydrologique, les reconnaissances géologiques et géotechniques (sondages, fouilles, essais en laboratoire), le dimensionnement hydraulique des ouvrages, le calcul de génie civil des ouvrages en béton armé et les pièces dessinées.

La réduction des délais a été obtenue également grâce à la maîtrise technique et à I'outil informatique.

Les travaux d'études ont été scindés en six missions distinctes : travaux topographiques, étude hydrologique, étude géologique et géotechnique, étude de faisabilité, étude d'exécution, et dossier d'appel d'offre. En effet l'étude hydrologique ne fait ressortir que les paramètres essentiels nécessaires à la suite des travaux d'études. L'étude géologique et géotechnique, sur la base 
d'une campagne de reconnaissance restreinte et d'essais en laboratoire limités, définit le type de remblai à mettre en place, les remèdes à prévoir dans le traitement des fondations, et les paramètres géotechniques rentrant dans le dimensionnement des ouvrages.

Le choix du type de remblai qui est lié à la disponibilité des matériaux de construction en quantité et en qualité, se limite à un remblai homogène ou zoné. Les détails du remblai font l'objet d'un plan d'exécution standard valable pour toutes les retenues, il suffit seulement de mettre les cotes correspondantes.

Le choix du type d'évacuateur de crues dépend essentiellement du relief. Il est préconisé des évacuateurs de crues à surface libre à entonnement frontal ou latéral. Les hauteurs des murs bajoyers ont été standardisées et des plans types de ferraillage de différentes hauteurs sont élaborés, prêts pour être adaptés à l'étude considérée. Les bassins d'amortissement qui sont préconisés sont de types USBR.

Deux types d'ouvrages de vidange et de prise d'eau ont fait l'objet d'une préparation technique et dimensionnelle, dans le but de les caler sur tous les sites de retenues collinaires, et des plans d'exécution sont élaborés et prêts à une large utilisation.

Pour mieux cerner ces études, un échantillon de plus d'une vingtaine de retenues collinaires réalisées et actuellement en exploitation, dans cinq Wilayas du Nord de l'Algérie, est présenté. La diffusion de notre expérience certes un peu courte comparativement à certains pays méditerranéens mérite d'être prise en considération et peut contribuer quelque part à améliorer la sécurité et les performances de ces ouvrages.

Mots clés : retenue collinaire, normalisation des études, dimensionnement hydraulique, conception des ouvrages, plans d'exécution, rationalisation.

\section{1 - INTRODUCTION}

Nous exposons dans cet article, la méthodologie d'approche dans la conception des retenues collinaires, adoptée en Algérie. En effet, l'expérience algérienne dans ce domaine a été acquise après quelques échecs enregistrés, suite à une mauvaise appréciation de l'importance et du contenu des études nécessaires à la réalisation de tels ouvrages.

\section{2 - HISTORIQUE}

Avec une démographie galopante et une demande en eau croissante, l'Algérie est contrainte de mobiliser au maximum ses ressources en eau superficielles par la réalisation d'un nombre assez important de retenues collinaires sur l'ensemble de son territoire (MHEF, 1986a, 1986b, 1987 ; EDF, 1989). Suite à de 
telles décisions, les premières administrations publiques concernées se sont lancées dans la construction de tels ouvrages sans pour autant se préoccuper des études préliminaires. Après 2 ans, l'échec d'un tel investissement fût au rendezvous avec des causes multiples telles que:

- Importance du bassin versant par rapport à la capacité de la cuvette avec un apport solide significatif entraînant un comblement rapide ;

- Engorgement de la cuvette face à un apport liquide très important lors des crues et sous dimensionnement de l'évacuateur entraînant la rupture de l'ouvrage ;

- Capacité trop importante de la cuvette face à des apports liquides faibles aboutissant à un non remplissage de la retenue ;

- Non identification de fondations perméables lors de la réalisation, entraînant des pertes par infiltrations considérables et une incapacité de stockage ;

- Mauvais compactage du remblai provoquant un tassement rapide entraînant la rupture de l'ouvrage par submersion.

Devant un tel échec, les décideurs se penchèrent sur le problème et prirent en charge sérieusement la conception de ces retenues, sur la base d'un dossier d'études répondant aux normes techniques (USDI BR, 1977). Malheureusement, le manque d'expérience dans de telles études a engendré un investissement colossal dans la réalisation des plans de conception qui souvent se sont avérés inutiles. Pour remédier à une telle situation, des concertations élargies à divers organismes (Ministère de l'Hydraulique et de la Pêche, Directions de l'Hydraulique de Wilaya, Bureaux d'études publics) se sont tenues, aboutissant à une normalisation des études de retenues.

\section{3 - MÉTHODOLOGIE ADOPTÉE}

En vue d'une normalisation dans la conception des études, 6 missions se sont dégagées.

La Mission 1 “Travaux topographiques" consistant en des levés de la cuvette et du site respectivement sur 40 ha (Échelle $1 / 2000^{\circ}$ ) et 10 ha (Échelle $\left.1 / 500^{e}\right)$;

La Mission 2 «Étude hydrologique » faisant ressortir la répartition intraannuelle des précipitations, de l'évaporation et des apports liquides, les hydrogrammes de crues probables (décennale et centennale) et les apports solides.

La Mission 3 «Étude géologique et géotechnique » consistant d'une part en une étude de la carte géologique et une visite de terrain et d'autre part en une campagne de reconnaissance des fondations et des matériaux de construction (zone d'emprunt). Aussi, dans la campagne de prospection, les nombres de sondages, de fouilles, d'échantillons à analyser en laboratoire et 
les linéaires sont rationalisés. Trois sondages sont implantés sur l'axe de la digue avec un linéaire par sondage égal à la hauteur de la digue, localisés un en thalweg et deux en rives.

Le nombre de puits à réaliser dans la cuvette est au maximum de six, avec une profondeur n'excédant pas $3 \mathrm{~m}$.

Si la géologie de surface est relativement bonne, les fouilles sont exécutées sur l'axe du barrage en remplacement des sondages notamment quand la hauteur du remblai n'excède pas $12 \mathrm{~m}$; toutefois, sur l'axe du remblai, la profondeur des fouilles peut être égale à $6 \mathrm{~m}$. Cette méthode d'investigation donne souvent de très bons résultats surtout quand le remblai est positionnée sur un cours temporaire (affluent primaire ou secondaire) et non pas sur le cours principal de l'oued.

Sur le cours principal d'un oued, les sondages sont inévitables, car la couche alluvionnaire perméable est beaucoup plus importante du fait que le substratum est beaucoup plus profond, comparativement à une fondation sur les cours d'eau temporaires,

Les essais d'eau ne sont pas obligatoirement nécessaires, vu leur coût très élevé alors que la perméabilité des fondations peut être appréciée au laboratoire à l'oedomètre et au pot de Terghazi respectivement pour les particules de diamètre inférieur et supérieur à $5 \mathrm{~mm}$. Les substratums dépassent rarement 6 $m$ de profondeur sur les cours d'eau temporaires notamment dans la partie Septentrionale de l'Algérie.

Pour les puits et fouilles relatifs aux zones d'emprunt, les profondeurs varient entre 1,5 et $3 \mathrm{~m}$ avec une prise d'une dizaine d'échantillons pour les essais en laboratoire portant sur l'identification du matériau et ses caractéristiques géotechniques (PHILIPONNAT, 1979). Ces essais consistent en une analyse granulométrique et en la détermination des limites d'Atterberg entrant dans la classification et l'identification du sol et au dimensionnement des filtres; en la réalisation de l'essai Proctor pour la définition de la densité apparente du sol et de la teneur en eau optimale ; en la réalisation de l'essai oedométrique pour la définition de la pression de consolidation, des coefficients de gonflement et de perméabilité et en la réalisation de l'essai de cisaillement pour la définition de l'angle de frottement interne et la cohésion de Casagrande.

La Mission 4 «Étude de faisabilité » consiste en la détermination des courbes topographiques (hauteur - surface - capacité), en une étude des simulations saisonnières tenant compte des apports, de l'évaporation, des besoins en eau (TOUAÏBIA, 1995), en considérant que les pertes par infiltration représentent 10 à $18 \%$ de l'apport moyen, en un calcul du laminage de crues sur la base de la crue centennale par la méthode de Kotchérine (IVANOV, 1979), en un dimensionnement du remblai (gabarit de l'ouvrage, revanche, hauteur, largeur en crête), en la protection des talus et en un calcul hydraulique de l'évacuateur de crues (crue centennale), de la vidange de fond et de la prise d'eau.

Pour des raisons pratiques et de sécurité, le type d'évacuateur est un déversoir à seuil libre, à entonnement frontal ou latéral placé à une des extrémités du remblai. Souvent, cet ouvrage est constitué d'un canal d'approche, d'un seuil, d'un chenal, d'un convergent, d'un coursier à pente unique et d'un bassin de dissipation de type USBR (USDI BR, 1977). 
L'ouvrage de vidange de fond et de prise d'eau (ouvrage combiné) est de type standard, le diamètre de la conduite est fixé à partir du temps d'une vidange rapide. La conduite de prise est solidaire de celle de la vidange avec un diamètre égal à la moitié de celle-ci.

Six plans guides sont élaborés permettant la définition des volumes des travaux approximatifs et sont livrés au client dans le dossier de faisabilité (plans de situation de l'aménagement à échelle $1 / 1000^{\circ}$ et celui des ouvrages à échelle $1 / 500^{\mathrm{e}}$, profils en long et en travers du remblai, profils en long de l'évacuateur de surface et de la vidange de fond).

La Mission 5 “Étude d'exécution ". Le projet d'exécution est composé du rapport explicatif et justificatif (note de calcul) complétant le rapport de synthèse (faisabilité) avec une étude d'infiltration, une étude des drains et filtres, un calcul de tassement et de stabilité des talus. Ce rapport est ponctué par un métré des volumes des travaux de réalisation de l'aménagement; des plans d'exécution avec des plans de situation détaillés de l'aménagement (échelle $1 / 1000^{\mathrm{e}}$ ) et des ouvrages (échelle $1 / 500^{\mathrm{e}}$ ); un profil en long du remblai avec tous les volumes des travaux relatifs à ce dernier avec 5 profils en travers (coupes) du remblai à différentes sections, un plan des détails du remblai (crête, drain, pied du talus amont); un profil en long et une vue en plan de l'évacuateur de crues avec tous les volumes des travaux relatifs à cet ouvrage ; des coupes de l'évacuateur de crues (plans de coffrage et de ferraillage); un profil en long et une vue en plan de la vidange de fond et des ouvrages de prise avec tous les volumes des travaux relatifs à cet ouvrage; des coupes de la vidange de fond, de l'ouvrage de tête et du puits régulateur (plans de coffrage et de ferraillage) ; des plans de terrassement ; un plan d'implantation des ouvrages par rapport aux stations géodésiques.

La Mission 6 «Dossier d'appel d'offre (DAO) " Ce dernier renferme, les règles financières et normes techniques, le détail des différents travaux à effectuer pour la construction des ouvrages, le volume des travaux à effectuer pour la réalisation de l'aménagement.

\section{4 - DISPOSITIONS DIMENSIONNELLES ET CONSTRUCTIVES}

\subsection{Le remblai}

Si le choix du type de remblai est lié à la disponibilité des matériaux de construction en quantité et en qualité, il se limite à un remblai homogène ou zoné.

Aussi, en dehors du calcul des filtres et de la stabilité des talus, les autres calculs s'avèrent abordables. En effet, pour le calcul des filtres, les log-sondages et les essais en laboratoire sur les échantillons peuvent montrer une hétérogénéité entre les matériaux des différentes fouilles se répercutant sur le meilleur choix à considérer de la courbe granulométrique. Pour la détermination du fuseau de la première couche de filtre, le concepteur, à partir des logsondages, écarte les courbes granulométriques extrêmes ; superpose celles 
qui se ressemblent sur un même système de coordonnées; choisit la courbe moyenne des courbes superposées, (celle du milieu); définit la méthode de calcul des filtres en fonction de l'allure de la courbe granulométrique de base qui peut être courbe uniforme et étroite ou courbe continue et étendue (POST et al., 1953).

Les granulométries ainsi définies sont respectées pour le drain de pied aval, sous les enrochements de protection du talus amont et pour le cas d'un remblai zoné comme couche de transition entre le noyau étanche et les recharges.

L'étude de la stabilité des talus nécessite la préparation minutieuse des données à inclure dans les calculs pour chaque cas de sollicitation.

Les détails du remblai (crête, berme aval avec le collecteur des eaux de pluies, drain aval, protection du talus amont) font l'objet d'un plan d'exécution standard, valable pour toutes les retenues; il suffit seulement de mettre les côtes correspondantes.

\subsection{L'évacuateur de crues}

Le choix du type d'un tel ouvrage dépend essentiellement de son emplacement (relief). Des évacuateurs à surface libre, latéral à entonnement frontal ou latéral sont préconisés.

La longueur déversante est définie en tenant compte d'une charge sur le déversoir comprise entre 0,8 et $1,2 \mathrm{~m}$ et de la quantité minimale de béton nécessaire pour la réalisation de l'ouvrage. A titre d'exemple, le prix d'un mètre cube $\left(1 \mathrm{~m}^{3}\right)$ de béton varie entre 15000 DA et 20000 DA et celui du mètre cube $\left(1 \mathrm{~m}^{3}\right)$ de remblai compacté entre $150 \mathrm{DA}-200 \mathrm{DA}$ soit 100 fois moins. Il y a lieu de noter, que pour un tel ouvrage, le calcul d'optimisation n'est pas nécessaire.

En plus du calcul hydraulique, les murs bajoyers doivent faire l'objet d'un calcul statique et de génie civil. À cet effet, un programme de calcul a été mis au point (Mur.xls) basé sur la méthode exposé par CHARON (1983).

Des hauteurs de murs (h) ont été standardisées et fixées à $22,53,03,5$ et $4 \mathrm{~m}$.

Les murs dépassant $4 \mathrm{~m}$ sont conçus avec des contreforts (Murc.xls). Des plans types de ferraillage des murs bajoyers de différentes hauteurs sont élaborés, prêts pour être adaptés à l'étude considérée.

Le bassin d'amortissement est de type USBR (USDI BR, 1977). Selon le nombre de Froude et la vitesse à la fin du coursier, le type I ou le type III est adopté. Le type II, de par sa complexité de réalisation est remplacé par le type III.

\subsection{Vidange de fond, prise d'eau et dérivation provisoire}

Deux types d'ouvrages de vidange ont fait l'objet d'une préparation technique et dimensionnelle, dans le but de les caler sur tous les sites de retenues collinaires. Le premier type est constitué d'une tour de prise à l'amont, d'une conduite en acier et d'un puits régulateur à l'aval de la retenue; le second type est constitué de l'ouvrage d'entrée, d'une conduite en acier et de la chambre 
des vannes. Des plans d'exécution (coffrage et ferraillage) sont élaborés et prêts pour une large utilisation.

La prise d'eau est assurée par piquage sur la conduite de vidange dans la chambre des vannes. Le diamètre de la conduite est égal à 0,5 fois celui de la vidange.

L'emplacement de l'ouvrage d'entrée a toujours causé un tracas aux concepteurs qui doit tenir compte du rôle joué par la dérivation provisoire lors de la construction de l'ouvrage et de l'utilisation de toute la quantité d'eau stockée, malgré le volume mort, correspondant aux dépôts de sédiments (volume mort qui peut dépasser un quart à un tiers de la capacité totale de la retenue). Pour assurer une longévité de la retenue supérieure à celle de l'étude, des barres d'attente sortant de l'ouvrage de tête sont prévues, permettant éventuellement une surélévation. La durée de vie de la retenue définissant la tranche morte reste toujours à l'appréciation du concepteur et varie de 10 à 20 ans. En effet, l'exemple de la retenue de Hammam dans l'Oranie, verrait son volume mort dépasser les $50 \%$ de la capacité à stocker $\left(216000 \mathrm{~m}^{3}\right.$ de vase pour un volume total de $400000 \mathrm{~m}^{3}$, si sa durée de vie est fixée à 20 ans).

Le tableau 1 résume quelques retenues réalisées (BENLAOUKLI, 1992, 1995 et 1998) et actuellement en exploitation.

Tableau 1 Identification de quelques retenues.

Table 1 Identification of several dams.

\begin{tabular}{llcccc}
\hline Nom & Wilaya & Hauteur $\mathbf{m}$ & $\begin{array}{c}\text { Volume mort } \\
\left(\mathbf{1 0}^{\mathbf{6}} \mathbf{m}^{\mathbf{3}}\right)\end{array}$ & $\begin{array}{c}\text { Capacité au } \\
\text { Niveau normal } \\
\left(\mathbf{1 0}^{\mathbf{6}} \mathbf{~}^{3} \mathbf{)}\right.\end{array}$ & $\begin{array}{c}\text { Durée de vie } \\
\text { an }\end{array}$ \\
\hline Lecbour & BBA & 17,8 & 0,25 & 0,9 & 20 \\
Hamada & BBA & 15,0 & 0,20 & 0,7 & 20 \\
Bou lardjem & Medea & 16,0 & 0,22 & 0,9 & 20 \\
El Hammam & Medea & 19,5 & 0,30 & 1,2 & 20 \\
Sidi Hamed & Blida & 17,0 & 0,25 & 1,2 & 20 \\
Amroussa & Blida & 15,8 & 0,13 & 0,6 & 20 \\
Merrakchi & Blida & 19,0 & 0,15 & 0,6 & 20 \\
Ben Yacoub & Djelfa & 15,5 & 0,30 & 1,2 & 15 \\
Kreireche* & Djelfa & 16,0 & 0,32 & 0,9 & 10 \\
Brouziane & Ain Defla & 16,8 & 0,25 & 0,74 & 20 \\
Djemaa & Ain Defla & 14,5 & 0,15 & 0,50 & 20 \\
\hline
\end{tabular}

* en construction. 


\section{5 - DÉLAIS D'EXÉCUTION DES ÉTUDES D'UNE RETENUE}

Les délais d'exécution d'une étude accordés dans les tous premiers marchés sont passés de 12 à 6 , voire 5 mois grâce à la maîtrise de la technique, à l'outil informatique et à la rationalisation du contenu des missions de l'étude. Nous résumons dans le tableau 2 le temps imparti à chaque partie de l'étude sur la base du constat de ces trois dernières années.

Tableau 2 Délai d'exécution en jours.

Table 2 Construction time (days).

\begin{tabular}{|c|c|}
\hline Désignation des travaux d'études & Délai d'exécution (jours) \\
\hline Travaux de topographie & 20 \\
\hline Étude* hydrologique & 07 \\
\hline $\begin{array}{l}\text { Étude géologique et géotechnique (y compris sondages, fouilles, } \\
\text { essais en laboratoire) }\end{array}$ & 60 \\
\hline $\begin{array}{l}\text { Étude de faisabilité proprement dite (courbes hauteur-surface- } \\
\text { capacité, régularisation saisonnière, laminage des crues, } \\
\text { dimensionnement de la digue, calcul hydraulique de l'évacuateur } \\
\text { de crues et de l'ouvrage de vidange et de prise, } 06 \text { plans guides) }\end{array}$ & 10 \\
\hline $\begin{array}{l}\text { Étude* d'exécution (infiltration, drain, stabilité, tassement, calcul } \\
\text { statique et de génie civil de l'évacuateur de crues, courbe } \\
\text { d'exploitation de la vidange de fond, } 16 \text { à18 plans d'exécutions) }\end{array}$ & 20 \\
\hline Dossier d'appel d'offres DAO (préalablement élaboré) & 02 \\
\hline
\end{tabular}

* étude programmable.

\section{6 - CONCLUSION}

Pendant quinze années, les retenues collinaires et les petits barrages ont fait l'objet de notre plus grande préoccupation. Tout au long de notre expérience, le travail s'est articulé sur les points les plus délicats. Si aujourd'hui, l'ingénieur arrive à concevoir un ouvrage de retenue au prix de 1200000 DA (25 000 \$) dans des délais inférieurs à 6 mois, c'est grâce à l'échange de l'information, aux concertations élargies et à l'outil informatique.

Plus de $80 \%$ de nos retenues sont fonctionnelles, les quelques échecs enregistrés nous permettent de mieux cerner quelques problèmes non identifiés entre les études et la réalité du terrain et qui restent liés beaucoup plus au facteur anthropique (surpâturage du talus aval, emportement et mise à nu des talus en gravier). Quelque soit l'étude de faisabilité d'une retenue que l'on entreprend, la fiabilité et la qualité de l'information et des données restent un souci majeur pour une meilleure estimation des apports liquide et solide et un meilleur traitement statistique impliqué dans toute prise de décision (TOUAIBIA, 2000). 


\section{RÉFÉRENCES BIBLIOGRAPHIQUES}

BENLAOUKLI B., 1992. Études d'exécutions des retenues collinaires de Lechbour, Tixter. Document interne. SETHYAL, Alger. $112 \mathrm{p}$.

BENLAOUKLI B., 1995. Études d'exécutions des retenues collinaires de Meloussa, R'horb, Djemaa, Bouziane. Document interne. Geosystem-BET, Alger. $222 \mathrm{p}$.

BENLAOUKLI B., 1998. Études d'exécutions des retenues collinaires El Bateun, Mdaourouch, Ain Sfa, Marguerite. Document interne. ENHPC, Alger.

CHARON P., 1983. Le calcul et la vérification des ouvrages en béton armé. Édition Eyrolles. Paris.

EDF., 1989. Séminaire sur la conduite des études de barrages. Document interne. Alger.

IVANOV A., NEGOVSKAIA T.,1979. Hydrologie et Régularisation des débits. Colosse Moscou.

Ministère de I'Hydraulique, de l'Environnement et des Forêts (MHEF), 1987. Guide maghrébin pour l'exécution des études et travaux de retenues collinaires. Alger.

Ministère de l'Hydraulique, de l'Environnement et des Forêts (MHEF), 1986a. Journées techniques sur les retenues collinaires. Document interne. Guelma. $27 \mathrm{p}$.

Ministère de l'Hydraulique, de l'Environnement et des Forêts (MHEF), 1986b. Séminaire national sur les retenues collinaires. Document interne. Sétif. $34 \mathrm{p}$.

PHILLIPONNAT G., 1979. Fondations et ouvrages en terre. Éditions Eyrolles. Paris.

POST G., Londe P., 1953 Les barrages en terre compactée. Pratiques américaines Gauthier-Villars. Paris.

USDI BR, United States Department of Interior. Bureau of Reclamation, 1977. Design of Small Dams. A water resources technical A publication. New York.

TOUAIBIA B, 1995 : Les Retenues. Polycope de cours de régularisation des débits. Ecole Nationale Supérieure de l'Hydraulique. Blida. Algérie. $60 \mathrm{p}$.

TOUAIBIA B, 2000. Érosion - Transport solide - Envasement de Barrage. Thèse de Doctorat d'État en Aménagement et Mise en Valeur. Institut National Agronomique d'El-Harrach. Alger. $180 \mathrm{p}$. 